\title{
Kindstötungen im 21. Jahrhundert: Erstmals Risikofaktoren identifiziert
}

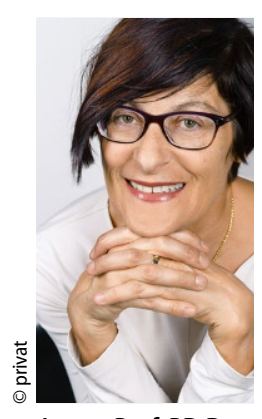

Assoc.-Prof. PD Dr.

Claudia Klier

Link: https://link springer.com/journal/ volumesAndlssues/737.

Quelle: Presseaussendung der MedUni-

Wien, 12.02.2019

\begin{abstract}
In einer nun im Themenheft der "Archives of Women's Mental Health" zum Thema „Kindstötung" publizierten Studie [1] konnte Claudia Klier von der Universitätsklinik für Kinder- und Jugendheilkunde der MedUni Wien erstmals gemeinsam mit finnischen Experten aus Helsinki und Turku Risikofaktoren von einmaligen und wiederholten Neugeborenentötungen identifizieren.,/Generell konnte gezeigt werden, dass wiederholte Neonatizide nicht so selten sind, wie angenommen. Im Zeitraum von 1995-2005 war überraschenderweise jeder dritte getötete Säugling auf einen vorher noch nicht entdeckten Wiederholungsfall zurückzuführen", sagt Claudia Klier, Leiterin der Pädiatrischen Psychosomatik an
\end{abstract}

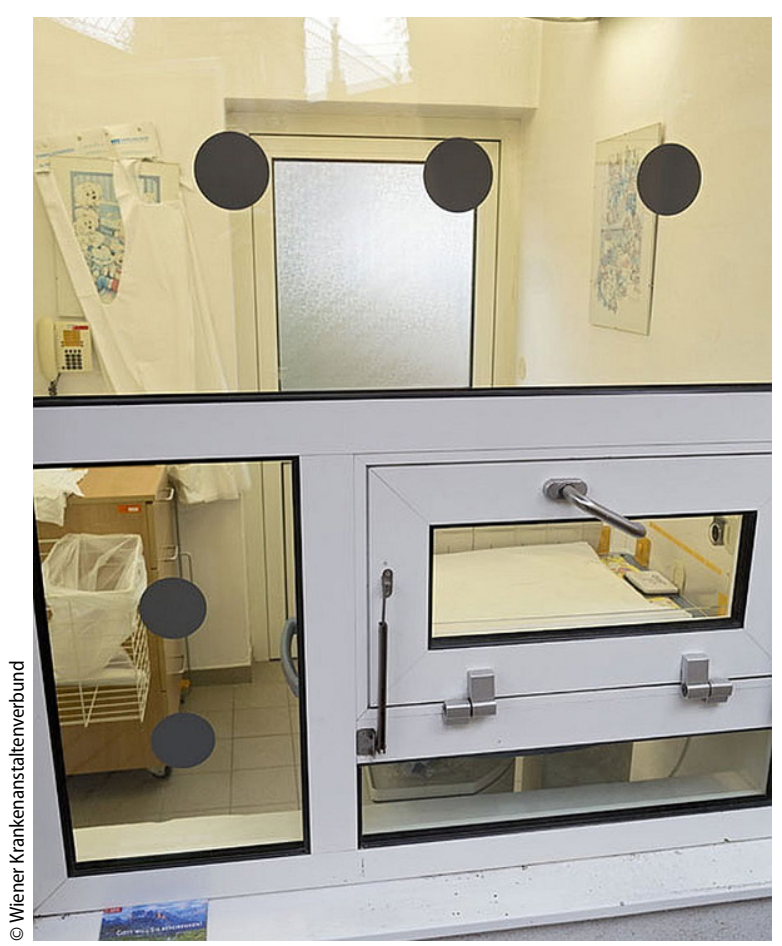

$\Delta$ Anonyme Geburt: Dieses Babynest befindet sich in 1160 Wien am Flötzersteig, neben dem Pavillon 40 der Kindernotfallambulanz des Wilhelminenspitals der Universitätsklinik für Kinder- und Jugendheilkunde der MedUni Wien (Foto).

\section{Was versteht man unter Neonatizid?}

Neonatizid ist die Tötung eines Kindes in den ersten 24 Stunden nach der Geburt. Der Tötung geht ein monatelanger Prozess von „Negierung“" voraus: Die Frau kann aufgrund verschiedener Traumata und/oder einer Persönlichkeitsstörung die Schwangerschaft nicht wahrhaben, negiert sie, und ihr soziales Umfeld hat - unabhängig von der Lebenssituation - häufig keine Kenntnis der Schwangerschaft. Eine Auseinandersetzung mit der ungewollten Schwangerschaft findet folglich nicht statt.

Klier: „Auch das Wort Schwangerschaft wird nicht benutzt. Die Frau erklärt bei Nachfragen die Gewichtszunahme durch zu viel Essen, Blähungen und andere Gründe, wodurch also eine Uminterpretation der Symptome stattfindet. Die Frauen haben keinen Kontakt zum Gesundheitssystem, die Geburt, von der die Frauen meist überrascht werden, erfolgt unassistiert und heimlich, was ein hohes Risiko für die Gebärende und das Kind birgt. Denn das Neugeborene wird entweder nicht versorgt oder aktiv getötet, da es in dieser Situation zu Panik und dissoziativen Zuständen bei der Gebärenden kommen kann.."

\section{Prävention ist möglich}

In Österreich ist die Zahl der Neugeborenentötungen stark rückläufig. Waren zwischen 1991 und 2001 noch rund sieben von 100.000 Neugeborenen betroffen, so sind es heutzutage nur mehr drei Kinder pro Jahr. Österreich liegt damit inzwischen im europäi- schen Mittelfeld, die skandinavischen Länder haben jedoch deutlich niedrigere Zahlen.

Zurückzuführen ist der Rückgang der Zahlen in Österreich auf die Einführung der „anonymen Geburt“ im Jahr 2002. Klier: „Die anonyme Geburt und Schwangerschaftsbegleitung ist ein sehr effektives Mittel, um diesen Frauen in ihrer schwierigen Situation zu helfen und sie vor, während und nach der Geburt medizinisch und psychosozial zu betreuen." Die anonyme Geburt ist an allen Abteilungen für Geburtshilfe in Österreich und einigen anderen europäischen Staaten möglich.

\section{Weitere Informationen:}

Das Forschungsteam hat eine Webpage mit allen wichtigen Informationen zum Thema für Betroffene, Fachleute und für die Teilnahme an laufenden Forschungsprojekten: http://anonymegeburt.at/anogeb-eur-map/

\section{Literatur}

1. Klier CM, Amon S, Putkonen $\mathrm{H}$, Fernandez Arias P, Weizmann-Henelius $G$ Repeated neonaticide: differences and similarities to single neonaticide events. Women's Health. https://doi.org/10.1007/s00737-018-0850-1

Paediatr. Paedolog. 2019 · 54:100 https://doi.org/10.1007/s00608 019-0671-0

(c) Springer-Verlag GmbH Austria, ein Teil von Springer Nature 2019 